\title{
Infecção parasitária de cordeiros e contaminação larval em pasto submetido à adubação nitrogenada ${ }^{1}$
}

\author{
Luiz Giovani de Pellegrini ${ }^{2}$, Ana Carolina R.S. de Pellegrin ${ }^{3}$, Alda Lúcia G. Monteiro ${ }^{4}$, \\ Marcelo B. Molento ${ }^{4}$, Mikael Neumann ${ }^{5}$ e Elaine Longhi ${ }^{6}$
}

\begin{abstract}
Pellegrini L.G., Pellegrin A.C.R.S., Monteiro A.L.G.,. Molento M.B. \& Longhi E. 2011. [Parasitic infection of lambs and larval contamination in pasture submitted to nitrogen fertilization.] Infecção parasitária de cordeiros e contaminação larval em pasto submetido à adubação nitrogenada. Pesquisa Veterinária Brasileira 32(12):10971101. Setor de Bovinocultura de Corte, Instituto Federal Farroupilha, Campus Júlio de Castilhos, São João do Barro Preto, Cx. Postal 38, Júlio de Castilhos, RS 98130-000, Brazil. E-mail: depellegrini@yahoo.com.br

The objective of this research was to evaluate the parasitic infection of lambs and larval contamination in pasture of ryegrass submitted to nitrogen fertilization. We used 72 weaned lambs that were distributed into 12 experimental units (paddocks) with six lambs (animal testing) in each one. The experimental design was randomized blocks, with four treatments ( $\mathrm{N}$ rates), with three replicates (paddocks). Regarding the number of eggs per gram of feces, there was no statistical difference between the different levels of nitrogen applied to the pasture. In relation to the number of recovered larvae from the pasture, there was a significant effect $(\mathrm{P}<0.05)$ through the linear decrease in accordance with the rates of nitrogen. No statistical difference was found when evaluating the distribution of larvae over the strata of the pasture. The interpretation of the stool culture revealed a predominance of the genera Haemonchus spp. and Trichostrongylus spp. independent of the dose of nitrogen applied.
\end{abstract}

INDEX TERMS: Dose of nitrogen, Haemonchus, larvae L3, sheep, strata.

RESUMO.- Objetivou-se avaliar a infecção parasitária de cordeiros e contaminação larval no pasto de azevém submetido a adubação nitrogenada. Foram utilizados 72 ovinos desmamados sendo estes distribuídos entre as 12 unidades experimentais (piquetes) com seis cordeiros

\footnotetext{
${ }^{1}$ Recebido em 1 de setembro de 2011.

Aceito para publicação em 28 de setembro de 2011.

${ }^{2}$ Setor de Zootecnia, Instituto Federal de Educação, Ciencia e Tecnologia Farroupilha Campus Júlio de Castilhos, São João do Barro Preto s/n, Interior, Cx. Postal 38, Júlio de Castilhos, RS 98130-000, Brasil.*Autor para correspondência: depellegrini@yahoo.com.br

${ }^{3}$ Programa de Pós-Graduação em Zootecnia, Universidade Federal de Santa Maria, Av. Roraima 1000, Camobi, Santa Maria, RS 97105-900, Brasil.

${ }^{4}$ Departamento de Medicina Veterinária, Universidade Federal do Paraná, Rua dos Funcionários 1540, Juvevê, Curitiba, PR 80035-050, Brasil.

${ }^{5}$ Departamento de Medicina Veterinária, Universidade Estadual do Centro-Oeste, Rua Simeão Camargo Varela de Sá 3, Cascavel, Guarapuava, PR 85040-080, Brasil.

${ }^{6}$ Departamento de Medicina Veterinária, Universidade Luterana do Brasil, Av. Eng ${ }^{\circ}$. Manfredo Barata Almeida da Fonseca 762, Jardim Aurélio Bernardi, Ji-Paraná, RO 76907-438, Brasil.
}

(animais testes) em cada. O delineamento experimental adotado foi o de blocos ao acaso, composto por quatro tratamentos (doses de N), com três repetições (piquetes). Em relação ao número de ovos de helmintos por grama de fezes não se obteve diferença estatística entre as diferentes doses de $\mathrm{N}$ aplicadas no pasto. Já em relação ao número de larvas recuperadas no pasto pode-se observar efeito significativo $(\mathrm{P}<0,05)$ através do comportamento linear decrescente de acordo com as doses de adubação nitrogenada. Não houve diferença estatística ao avaliar a distribuição das larvas ao longo dos estratos do pasto. A interpretação da coprocultura revelou maior predominância de Haemonchus spp. e Trichostrongylus spp. independente da dose de nitrogênio aplicada.

TERMOS DE INDEXAÇÃO: Doses de nitrogênio, estratos, Haemonchus, larvas $\mathrm{L}_{3}$, ovinos.

\section{INTRODUÇ̃̃o}

A produção de ovina é crescente nos últimos anos, com produtores focados na busca do aumento da produtividade e no aproveitamento dos mais variados produtos fornecidos por 
esta espécie. Contudo, o parasitismo gastrointestinal é responsável por grande parte dos prejuízos observados nas diversas criações destes pequenos ruminantes, reduzindo consequentemente o potencial produtivo (Molento et al. 2004).

Esses prejuízos causados são ainda mais acentuados quando os ovinos são mantidos em pastejo, devido ao ciclo de desenvolvimento das larvas, as altas lotações das áreas e ao manejo inadequado dos animais e das pastagens (Dittrich et al. 2004). Realidade esta que vem sendo vivenciada na região Sul do Brasil, pois ainda na grande maioria das propriedades o sistema de produção de cordeiros para o abate é a pasto (Pellegrin et al. 2009). Neste sentido, Macedo et al. (2000) afirmam que o ganho de peso de cordeiros em terminação a pasto é limitado pela verminose; sendo os cordeiros desmamados a categoria mais acometida (Echevarria et al. 1989).

Na busca pela intensificação da produção, a adubação nitrogenada é uma das práticas de manejo utilizadas para proporcionar maior lotação nas áreas, aumentando a capacidade de suporte do pasto e o ganho por área. Contudo, deve-se considerar que a quantidade de nitrogênio utilizado pode alterar a estrutura da vegetação mesmo se esta for manejada a uma mesma altura de pastejo (Pellegrini et al. 2010). Porém, Pegoraro et al. (2008) afirmam que ainda há muitas controvérsias a respeito de como o manejo da pastagem influência a carga parasitária em ovinos.

Estratégias de manejo da pastagem, visando à redução da ingestão de larvas infectantes (L3) pelos animais, são essenciais para o controle dos nematóides gastrintestinais, assim como o conhecimento detalhado da dinâmica da população e da localização das larvas infectantes na pastagem (Rocha et al. 2007).

Desta maneira, objetivou-se avaliar a infecção parasitária de cordeiros e contaminação larval no pasto de azevém submetido a adubação nitrogenada.

\section{MATERIAL E MÉTODOS}

A pesquisa foi realizada na Universidade Estadual do Centro-Oeste, em Guarapuava-Paraná. O clima da região é o Cfb (Subtropical Mesotérmico Úmido), sem estação seca, com verões frescos e inverno moderado conforme a classificação de Köppen, em altitude de aproximadamente 1.100 m, precipitação média anual de 1.944 $\mathrm{mm}$, temperaturas médias anuais mínima de $12,7^{\circ} \mathrm{C}$ e máxima de $23,5^{\circ} \mathrm{C}$ e umidade relativa do ar de $77,9 \%$.

0 experimento foi instalado em área tradicionalmente produtora de grãos (verão e inverno). No ano do presente experimento passou a ser área de integração lavoura-pecuária, sendo o primeiro ano a ser estabelecida uma pastagem temperada para pastejo com ovinos. Neste sentido, considerada como área livre de parasitas. Sendo assim, o pasto de azevém (Lolium multiflorum Lam.) foi implantado em 06 de junho de 2006, em sistema de plantio direto, após aplicação do herbicida gliphosate $(360 \mathrm{~g} / \mathrm{L})$. No plantio do azevém, o espaçamento entre linhas foi de $15 \mathrm{~cm}$, a profundidade de semeadura foi de $0,4 \mathrm{~cm}$ e a densidade de semeadura foi de 45 $\mathrm{kg}$ de sementes/ha.

Os tratamentos consistiram das doses 0, 75, 150 e $225 \mathrm{~kg}$ de nitrogênio $(\mathrm{N}) /$ ha, na forma de uréia $(45 \%$ de $\mathrm{N})$. Procedeu-se à adubação de fundação quando foi realizada a semeadura da pastagem com $60 \mathrm{~kg} \mathrm{P}_{2} \mathrm{O}_{5} /$ ha e $60 \mathrm{~kg} \mathrm{~K} 2 \mathrm{O} / \mathrm{ha}$, conforme Comissão de Fertilidade do Solo RS/SC (1995). Após 35 dias da semeadura (11 de julho de 2006), correspondendo ao início do perfilhamento das plantas, realizou-se a aplicação da adubação nitrogenada em cobertura na forma de uréia, em dose única.

A entrada dos animais na área experimental ocorreu no dia 20 de agosto de 2006, o que corresponde a um período de 40 dias após a aplicação das doses de N. O experimento teve duração de 90 dias, sendo seis dias para adaptação dos animais ao pasto e às instalações e 84 dias para avaliação. A área total do experimento foi de 3,1 ha, sendo 0,5 ha destinados à manutenção de animais reguladores e 2,64 ha subdivididos em três blocos com 0,88 ha; cada bloco foi dividido em quatro piquetes com 0,22 ha.

Foram utilizados 72 ovinos desmamados, com idade média de dois meses e peso vivo inicial de $24,7 \pm 0,57 \mathrm{~kg}$, distribuídos aleatoriamente nos tratamentos de acordo com peso e sexo. Cada lote foi constituído por dois ovinos machos castrados, dois machos não castrados e duas fêmeas.

Os animais foram distribuídos entre as 12 unidades experimentais (piquetes) com seis cordeiros (animais testes) em cada, sendo estes mantidos em azevém em sistema de lotação contínua com carga variável. A lotação contínua foi adotada por intermédio da técnica put-and-take (Moot \& Lucas 1952), sendo utilizando os cordeiros testes e número variável de reguladores por piquete, com o objetivo de manter a altura do pasto de $14-15 \mathrm{~cm}$, seguindo recomendações de Pontes et al. (2004). Os ajustes da lotação foram feitos em intervalos de três dias, considerando-se a relação entre altura e massa de forragem, segundo Carvalho et al. (2001).

A infecção parasitária no animal foi determinada pela contagem de ovos por grama de fezes (OPG) utilizando-se a técnica de Gordon \& Withlock (1939) modificada. Posteriormente, foi realizada a cultura de fezes pelo Método de Roberts e O’Sullivan (Ueno \& Gonçalves 1998) para identificação dos gêneros presentes nas fezes.

Para a avaliação da infestação do pasto, foram coletadas cinco amostras por piquete, em pontos aleatórios, com auxilio de um quadro com área de $0,0625 \mathrm{~m}^{2}(25 \times 25 \mathrm{~cm})$. As coletas foram realizadas nas horas mais amenas da manhã (das 7h30min às 10h), período em que, segundo Camuset (1994) e Araújo (2009) haveria maior movimentação vertical das larvas (fototropismo). Para avaliar a presença de larvas nas diferentes alturas da forragem, o corte do pasto foi estratificado em três camadas. 0 corte começou no estrato superior (acima de $10 \mathrm{~cm}$ ), e em seguida foram realizados os cortes sucessivamente nos extratos de $5-10 \mathrm{~cm}$ e $0-5 \mathrm{~cm}$. As amostras foram acondicionadas em sacos plásticos previamente identificados, mantidas refrigeradas em caixa térmica e posteriormente em refrigerador à temperatura média de $10^{\circ} \mathrm{C}$, por período máximo de 48 horas, e em seguida encaminhadas aos Laboratório de Parasitologia da Unicentro e da UFPR para o processamento de acordo com as técnicas de Taylor (1939) e Raynauld \& Gruner (1982).

0 delineamento experimental adotado foi o de blocos ao acaso, composto por quatro tratamentos (doses de N), com três repetições (piquetes). Os dados coletados para cada variável foram submetidos à análise de variância a $5 \%$ de significância, sendo os resultados significativos submetidos à análise de regressão para níveis e para os demais a comparação de medias pelo teste Tukey, por intermédio do programa estatístico SAS (2004).

\section{RESULTADOS E DISCUSSÃO}

0 pasto foi manejado a uma altura média de $17,7 \mathrm{~cm}$ independente da dose de nitrogênio (N) aplicada, sendo assim, obtiveram-se estruturas do pasto diferenciadas pela massa da forragem e massa de lâminas foliares (Quadro 1). Ainda obteve-se comportamento linear crescente da carga animal com o aumento da dose de $\mathrm{N}$ aplicada (Quadro 1).

Em relação ao número de ovos de helmintos por grama de fezes não se obteve diferença estatística entre as dife- 
Quadro 1. Altura, massa de forragem (MF), massa de lâminas foliares (MLF), densidade, oferta de forragem ( $\mathrm{kg}$ massa de forragem $/ 100 \mathrm{~kg} \mathrm{PV}$ ), ganho de peso médio diário (GMD), carga animal (CAN) do pasto de azevém sob pastejo contínuo de cordeiros de corte, em função das diferentes doses de nitrogênio

\begin{tabular}{|c|c|c|c|c|c|}
\hline \multirow[t]{2}{*}{ Parâmetros } & \multicolumn{4}{|c|}{ Doses de nitrogênio (kg/ha) } & \multirow[t]{2}{*}{ Equação de Regressão } \\
\hline & 0 & 75 & 150 & 225 & \\
\hline Altura, cm & 16,0 & 18,4 & 18,3 & 18,2 & $\begin{array}{l}\mathrm{Y}=17,7 \\
(\mathrm{R} 2: 0,5169 ; \mathrm{CV}: 7,8922 \% ; \mathrm{P}=0,3796)\end{array}$ \\
\hline MF, kg de MS/ha & 1837,4 & 2103,5 & 2084,0 & 2549,4 & $\begin{array}{l}Y=1826,0950+2,8219 N \\
(R 2: 0,2145 ; C V: 21,5812 \% ; P=0,0009)\end{array}$ \\
\hline MLF, kg de MS/ha & 515,0 & 599,7 & 662,6 & 814,6 & $\begin{array}{l}Y=503,7041+1,2824 \mathrm{~N} \\
(R 2: 0,0699 ; \mathrm{CV}: 61,8352 \% ; P=0,0694)\end{array}$ \\
\hline Oferta de Forragem & 19,1 & 14,7 & 12,9 & 13,2 & $\begin{array}{l}Y=19,0950-0,0731 N+0,0002 N^{2} \\
(R 2: 0,7039 ; C V: 12,4522 \% ; P=0,0042)\end{array}$ \\
\hline GMD, kg PV/ha/dia & 0,118 & 0,133 & 0,134 & 0,147 & $\begin{array}{l}Y=0,133 \\
(R 2: 0,3670 ; C V: 18,1730 \% ; P=0,5751)\end{array}$ \\
\hline CAN, kg PV/ha/dia & 770,1 & 1095,7 & 1252,2 & 1469,5 & $\begin{array}{l}Y=808,6833+3,0062 N \\
(R 2: 0,8290 ; C V: 10,9341 \% ; P=0,0001)\end{array}$ \\
\hline
\end{tabular}

Quadro 2. Número de ovos por grama de fezes (OPG) e número total de larvas L3 recuperadas no pasto de azevém sob pastejo contínuo de cordeiros de corte, em função das diferentes doses de nitrogênio

\begin{tabular}{|c|c|c|c|c|c|}
\hline \multirow[t]{2}{*}{ Parâmetros } & \multicolumn{4}{|c|}{ Doses de nitrogênio (kg/ha) } & \multirow[t]{2}{*}{ Equação de Regressão } \\
\hline & 0 & 75 & 150 & 225 & \\
\hline$\overline{\mathrm{OPG}}$ & 1621 & 1140 & 1121 & 1074 & $\begin{array}{l}Y=1239 \\
(R 2: 0,3463 ; C V: 38,3026 \% ; P=0,6821)\end{array}$ \\
\hline Larvas, L3/kg MS & 23 & 11 & 5 & 4 & $\begin{array}{l}Y=0,0293-0,0842 N \\
(R 2: 0,1958 ; C V: 37,6580 \% ; P=0,0069)\end{array}$ \\
\hline
\end{tabular}

rentes doses de $\mathrm{N}$ aplicadas no pasto, apresentando valor médio de 1239 (Quadro 2). Já em relação ao número de larvas recuperadas no pasto pode-se observar efeito significativo $(\mathrm{P}<0,05)$ através do comportamento linear decrescente de 0,8 larvas para cada $10 \mathrm{~kg}$ de $\mathrm{N}$ aplicado no pasto (Quadro 2). A utilização de N nas doses de 0, 75, 150 e 225 $\mathrm{kg}$ resultou, respectivamente, em porcentagens de larvas no pasto na ordem de 52,2, 78,5 e 82,8 \% inferiores a não utilização de N.

A diminuição no número de larvas com o aumento da dose de $\mathrm{N}$ pode ser justificado em função do arranjo espacial do pasto. Apesar do pasto de azevém ter sido manejado a mesma altura nas diferentes doses, o aumento de $\mathrm{N}$ aplicado proporcionou aumento na massa de forragem e consequentemente aumento na carga animal, o que resultou em redução na oferta na medida em que se elevou a dose. Este comportamento do pasto associado ao comportamento do pastejo dos animais pode ter proporcionado um microclima menos favorável ao desenvolvimento das larvas no pasto. Corroborando com os resultados encontrados neste experimento Pegoraro et al. (2008) trabalhando com cordeiros mantidos em pasto de azevém também observaram maior recuperação de larvas onde havia maior oferta de forragem.

Ao avaliar a distribuição das larvas ao longo dos estratos do pasto observa-se que não houve diferença estatística, com valores médios de 8,7, 15,6 e 7,9 larvas para o estrato de $0-5 \mathrm{~cm}, 5-10 \mathrm{~cm}$ e acima de $10 \mathrm{~cm}$, respectivamente (Quadro 3). Esses valores demonstram que 27,1\% das larvas estão no estrato inferior, $48,7 \%$ no estrato intermediário e $24,2 \%$ no estrato superior. Contrariamente ao observado no presente estudo Vlassof (1982) e Pegoraro et al. (2008) relatam que a maior concentração de larvas infectantes esta na parte inferior da planta, esses autores observaram que $80 \%$ das larvas infectantes localizavam-se nos primeiros $5 \mathrm{~cm}$ do relvado em pasto de azevém.

Colaborando com os resultados expressos Moss \& Vlassoff (1993) observaram que $28,1 \%$ das larvas encontravam-se na base da planta, $38,1 \%$ entre $2,6-7,5 \mathrm{~cm}$ de altura, $14,9 \%$ entre $7,6-12,5 \mathrm{~cm}$ e $18,8 \%$ acima de $12,5 \mathrm{~cm}$. E ainda Dittrich et al. (2004) observaram que pasto de azevém manejado a uma altura de $44 \mathrm{~cm}$ apresentam maior porcentagem de larvas infectantes no estrato superior quando comparado a pasto de azevém manejado a $82 \mathrm{~cm}$. Isso significa dizer que a localização das larvas infectantes nos diferentes estratos pode ser influenciada por vários fatores, sendo um dos fatores determinantes o manejo do pasto, em especial a oferta de forragem que altera o microclima, reforçando o conceito de Pellegrini et al. (2010) de que a altura não é o único fator que deve ser observado no momento do manejo da pastagem.

Ainda vale ressaltar que apesar da maior concentração de larvas $(48,7 \%)$ no estrato intermediário não houve inter-

Quadro 3. Número total de larvas L3, por estrato, recuperadas no pasto de azevém sob pastejo contínuo de cordeiros de corte, em função das diferentes doses de nitrogênio

\begin{tabular}{cccccc}
\hline Estrato & \multicolumn{3}{c}{ Doses de nitrogênio (kg/ha) } & \multirow{2}{*}{ Média } \\
\cline { 2 - 4 } & 0 & 75 & 150 & 225 & \\
\hline $0-5 \mathrm{~cm}$ & 20,0 & 7,8 & 4,1 & 3,0 & $8,7 \mathrm{a}$ \\
$5-10 \mathrm{~cm}$ & 40,7 & 14,1 & 4,8 & 3,0 & $15,6 \mathrm{a}$ \\
Acima de $10 \mathrm{~cm}$ & 8,1 & 11,1 & 5,9 & 6,3 & $7,9 \mathrm{a}$
\end{tabular}

Médias, seguidas de letras minúsculas iguais na linha não diferem entre si pelo teste Tukey ao nível de significância de $5 \%$. 
ferência no desempenho dos animais (Quadro 1). Isso porque independente da dose de $\mathrm{N}$ aplicada os animais tiveram a possibilidade de exercer a seletividade, visto que as ofertas estavam acima do que é preconizado pelo NRC (2007). Outro ponto relevante é o fato dos animais terem sido manejados no pasto com altura média de $17,7 \mathrm{~cm}$, onde Carvalho et al. (1999) afirmam que o estrato pastejado pelos ovinos corresponde, preferencialmente, a aproximadamente $50 \%$ da porção superior da planta, sendo assim muito pouco do estrato intermediário foi pastejado pelos animais.

A interpretação da coprocultura revelou maior predominância de Haemonchus spp. e Trichostrongylus spp. independente da dose de nitrogênio aplicada. A participação de Haemonchus spp. foi de 43, 52, 53 e $47 \%$ e a de Trichostrongylus spp. de 37, 40, 32 e 34\%, respectivamente, para as doses $0,75,150$ e $225 \mathrm{~kg} /$ ha de $\mathrm{N}$ (Quadro 4).

Quadro 4. Porcentagem da espécie de helminto no pasto de azevém sob pastejo contínuo de cordeiros de corte, em função das diferentes doses de nitrogênio

\begin{tabular}{ccccc}
\hline $\begin{array}{c}\text { Doses de N } \\
\text { (kg/ha) }\end{array}$ & \multicolumn{4}{c}{ Percentual de helmintos (\%) } \\
\cline { 2 - 5 } & $\begin{array}{c}\text { Haemonchus } \\
\text { spp. }\end{array}$ & $\begin{array}{c}\text { Trichostrongylus } \\
\text { spp. }\end{array}$ & $\begin{array}{c}\text { Ostertagia } \\
\text { spp. }\end{array}$ & $\begin{array}{c}\text { Cooperia } \\
\text { spp. }\end{array}$ \\
\hline 0 & 43 & 37 & 5 & 2 \\
75 & 52 & 40 & 7 & 1 \\
150 & 53 & 32 & 5 & 3 \\
225 & 47 & 34 & 8 & 4
\end{tabular}

Entre os dois gêneros de maior participação, constatou-se maior prevalência de Haemonchus spp. no pasto $(90,5 \%)$, bem como, nos animais $(58 \%)$ independente da dose de $\mathrm{N}$ aplicada (Quadro 5 ). Esse fato deve-se às condições climáticas ocorridas durante o experimento (Fig.1) e ao potencial biótico. As condições climáticas favoreceram 0 desenvolvimento do gênero Haemonchus, onde durante o período experimental as temperaturas foram amenas, média de $17,1^{\circ} \mathrm{C}$, e umidade elevada $(70,8 \%)$.

Com relação ao potencial biótico, que segundo Pegoraro et al. (2008) é definido como a capacidade de multiplicação em razão do tempo, onde Haemonchus spp. apresentam capacidade bastante elevada, com oviposição diária entre 5.000 e 10.000 ovos, muito superior ao de Trichostrongylus spp., que é de 100 a 200 ovos por dia (Romero \& Boero 2001). Este fato justifica a elevada proporção da participação de Haemonchus spp. nos animais e consequentemente no pasto.

Quadro 5. Percentagem do total de larvas L3 de Haemonchus spp. e de Trichostrongylus spp. recuperadas no pasto de azevém e da avaliação de coprocultura, em função das diferentes doses de nitrogênio

\begin{tabular}{cccccc}
\hline Parasitas & \multicolumn{4}{c}{ Doses de N (kg N/ha) } & Média \\
\cline { 2 - 4 } & 0 & 75 & 150 & 225 \\
\cline { 2 - 4 } & \multicolumn{4}{c}{ Pasto (\%) } \\
\cline { 2 - 4 } Haemonchus spp. & 93,0 & 85,7 & 91,5 & 91,7 & 90,5 \\
Trichostrongylus spp. & 7,0 & 14,3 & 8,5 & 8,3 & 9,5 \\
& \multicolumn{4}{c}{ Coprocultura (\%) } \\
Haemonchus spp. & 54,2 & 57,1 & 62,4 & 58,4 \\
Trichostrongylus spp. & 45,8 & 42,9 & 37,6 & 41,6 & 42,0
\end{tabular}

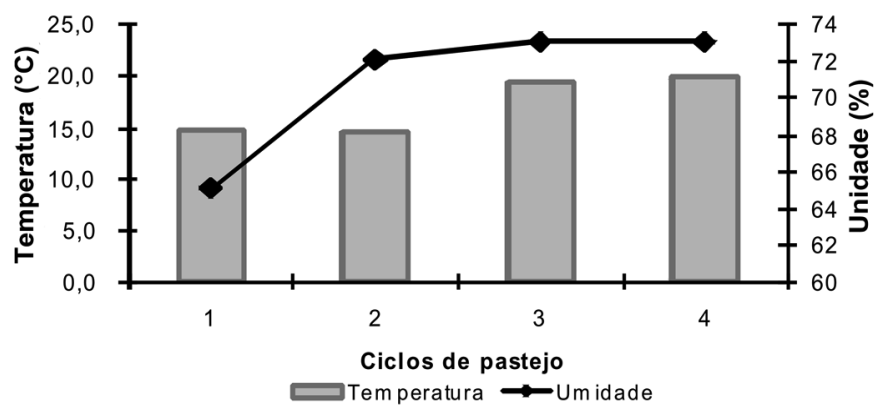

Fig.1. Valores médios de temperatura $\left({ }^{\circ} \mathrm{C}\right)$ e umidade relativa do ar (\%) ao longo dos ciclos de pastejo.

\section{CONCLUSÕES}

O manejo do pasto a uma mesma altura, mas com aumento na dose de nitrogênio, proporcionou redução no número de larvas recuperadas no pasto.

0 aumento na oferta de forragem ocasionou aumento no número de larvas recuperadas no pasto.

Independente da dose de nitrogênio, mas desde que as condições sejam favoráveis, o principal parasita encontrado no pasto e nos animais é Haemonchus spp.

Ainda há a necessidade de mais estudos com relação a influencia do manejo do pasto e do pastejo na carga parasitária dos animais e do pasto.

\section{REFERÊNCIAS}

Araújo F.B. 2009. Controle de nematóides gastrintestinais de ovinos com o uso do fungo nematófago Duddingtonia flagrans. Dissertação de Mestrado em Parasitologia Animal, Departamento Medicina Veterinária, Setor de Ciências Agrárias, Universidade Federal do Paraná, Curitiba, PR. $115 p$.

Camuset P. 1994. Epidemiologie des strongyloses: Parasitisme des bovins. Bulletin des GTV n.e., p.27-42.

Carvalho P.C.F., Marçal G.K., Ribeiro Filho H.M.N., Poli C.H.E.C., Trindade J.K., Oliveira J.R.O., Nabinger C. \& Moraes A. 2001. Pastagens altas podem limitar o consumo dos animais. Anais 39a Reunião Anual da Sociedade Brasileira de Zootecnia, Piracicaba, p.265-268.

Carvalho P.C.F., Prache S., Roguet C. \& Louault F. 1999. Defoliation process by ewes of reproductive compared to vegetative swards. Proc. $4^{\text {th }}$ International Symposium on the Nutrition of Herbivores, San Antonio.

Comissão de Fertilidade do Solo RS/SC 1995. Recomendações de Adubação e Calagem para os Estados do Rio Grande do Sul e Santa Catarina. $3^{a}$ ed. Sociedade Brasileira de Ciência do Solo/Embrapa-CNPT, Passo Fundo. 223p.

Dittrich J.R., Gazda T.L., Piazzetta R.G., Rodrigues C.S., Oikawa M.G. \& Soccol V.T. 2004. Localização de larvas L3 de helmintos gastrointestinais de ovinos nasplantas forrageiras: efeito da altura e da espécie vegetal. Archs Vet. Sci. 9(2):43-48.

Echevarria F.A.M., Pinheiro A.C. \& Corrêa M.B.C. 1989. Controle estratégico da verminose ovina no Rio Grande do Sul. $2^{\circ}$ Curso de Parasitologia Animal. CBPV, Bagé, p.159-163.

Gordon H.M.C.L. \& Whitlock H.V. 1939. A new tecnique for counting nematode eggs in sheep faeces. J. Counc. Sci. Indian Res. 12:50-52.

Macedo F.A.F., Siqueira E.R. \& Martins E.N. 2000. Análise econômica da produção de carne de cordeiros sob dois sistemas de terminação: pastagem e confinamento. Ciência Rural 30(4):677-680.

Molento M.B., Tasca C., Gallo A., Ferreira M., Bononi R. \& Stecca E. 2004. Método Famacha ${ }^{\circledR}$ como parâmetro clínico individual de infecção por Haemonchus contortus em pequenos ruminantes. Ciência Rural 34(4):11391145 . 
Moot G.O. \& Lucas H.L.1952. The design conduct and interpretation of grazing trials on cultivated and improved pastures. Proc. $6^{\text {th }}$ International Grassland Congress, State College, Pensylvania, p.1380-1395.

Moss R.A. \& Vlassoff A. 1993. Effect of herbage species on gastro-intestinal roundworm populations and their distribution. N. Z. J. Agric. Res. 36:371-375.

NRC 2007. Nutrients Requeriments of Small Ruminants. National Research Council, Washington, DC. 362p.

Pegoraro E.J., Poli C.H.E.C., Carvalho P.C.F., Gomes M.J.T.M. \& Ficher V. 2008. Manejo da pastagem de azevém, contaminação larval no pasto e infecção parasitária em ovinos. Pesq. Agropec. Bras. 43(10):1397-1403.

Pellegrin A.C.R.S., Pellegrini L.G., Monteiro A.L.G., Molento M.B., Neumann M. \& Pellegrini L.G. 2009. Comportamento de parasitas gastrointestinais dos ovinos em pastagem de azevém anual (Lolium multiflorum Lam.) submetida a diferentes doses de nitrogênio. Anais XIV Simpósio Paranaense de Ovinocultura, II Simpósio Paranaense de Caprinos, II Simpósio Sul Brasileiro de Ovinos e Caprinos, Curitiba, PR, p.1-3.

Pellegrini L.G., Monteiro, A.L.G., Neumann M., Moraes A., Pellegrin A.C.R.S. \& Lustosa S.B.C. 2010. Produção e qualidade de azevém-anual submetido à adubação nitrogenada sob pastejo por cordeiros. Revta Bras. Zootec. 39(9):1894-1904.
Pontes L.S., Nabinger C., Carvalho P.C.F. \& Soares A.B. 2003. Fluxo de biomassa em pastagem de azevém anual (Lolium multiflorum Lam.) manejada em diferentes alturas. Revta Bras. Zootec. 33:529-537.

Raynauld J.P. \& Gruner L. 1982. Feasibility of herbage sampling in large extensive pastures and availability of cattle nematode infective larvae in mountain pastures. Vet. Parasitol.10:57-64.

Rocha R.A., Bricarello P.A., Rocha G.P. \& Amarante A.F.T. 2007. Recuperação de larvas de Trichostrongylus colubriformis em diferentes estratos de Brachiaria decumbens e Panicum maximum. Revta Bras. Parasitol. Vet. 16(2):77-82.

Romero J.R. \& Boero C.A. 2001. Epidemiología de la gastroenterites verminosa de los ovinos em las regiones templadas y cálidas de la Argentina. Analecta Veterinaria 21:21-37.

SAS 2004. SAS/STAT User's Guide. SAS- Statistical Analisys System Institute Inc., Cary, NC. 5135p.

Taylor E.L. 1939. Technique for the estimation of pastures infestation by strongyle larvae. Parasitology 31:473-478.

Ueno H. \& Gonçalves V.C. 1998. Manual para Diagnóstico das Helmintoses de Ruminantes. 4 a ed. Tóquio: Japan International Cooperation Agency, 145p.

Vlassof A.1982. Biology and population dynamics of the free-living stages of gastrointestinal nematodes of sheep, p.11-20. In: Ross A.D. (Ed.), Control of Internal Parasites in Sheep. Lincoln College, New Zealand. 